\title{
Tidal dissipation in stars and fluid planetary layers and its impact on the evolution of star-planet systems
}

\author{
P. Auclair-Desrotour ${ }^{1,2, a}$, S. Mathis ${ }^{2, b}$, and C. Le Poncin-Lafitte ${ }^{3, c}$
}

1 IMCCE, Observatoire de Paris, UMR 8028 du CNRS, UPMC, 77 Av. Denfert-Rochereau, 75014 Paris, France

2 Laboratoire AIM Paris-Saclay, CEA/DSM - CNRS - Université Paris Diderot, IRFU/SAp Centre de Saclay, F-91191 Gif-sur-Yvette Cedex, France

3 SYRTE, Observatoire de Paris, UMR 8630 du CNRS, UPMC, 77 Av. Denfert-Rochereau, 75014 Paris, France

\begin{abstract}
Tidal dissipation in stars and planets is one of the key physical mechanisms that drive the evolution of planetary systems. It intrinsically depends on the nature of the tidal response of celestial bodies, which is directly linked to their internal structure and friction. Indeed, it is highly resonant in the case of fluids. In this work, we present a local analytical modeling of tidal gravito-inertial waves, which can be excited in stars and fluid planetary layers. This model allows us to understand the properties of their resonant dissipation as a function of the excitation frequencies, the rotation, the stratification, and the viscous and thermal properties of the studied fluid regions. Next, we introduce such a complex tidal dissipation frequency-spectra in a celestial mechanics numerical code to give a qualitative overview of its impact on the evolution of planetary systems. We consider the example of a two-body coplanar system with a punctual perturber orbiting a central fluid body. We demonstrate how the viscous dissipation of tidal waves can lead to a strongly erratic orbital evolution. Finally, we characterize such a non-regular dynamics as a function of the properties of resonances, which have been determined thanks to our local fluid model.
\end{abstract}

\section{Introduction}

Tidal dissipation in stars and planets is one of the key physical mechanisms that drive the evolution of planetary systems. In this context, [1] introduced the tidal quality factor $Q$, which is a general way to parametrize its strength in celestial mechanics. This dissipation is of great importance since it drives the secular orbital/spin evolution of stars, planets and satellites by converting the kinetic energy of tides into internal heating [2,3]. However, the mechanisms driving tidal dissipation are not the same in solids and fluids. Indeed, [4,5] show that the $Q$ factor of solid bodies varies smoothly with the forcing frequency. This is not the case of fluid regions, in which a resonant response to tidal perturbations is obtained [6-9]. It leads to a strong variation of the $Q$ factor as a function of the tidal frequency because of the viscous friction and thermal diffusion acting on fluid tidal waves and to an erratic evolution of the orbital dynamics [10].

This work focuses on the viscous friction applied on low-frequency tidal gravito-inertial waves. They constitute the tidal response of stars, of the external envelope of giant planets and of the fluid layers of rocky planets and satellites. Inertial waves are driven by rotation, gravity waves by strati-

\footnotetext{
a e-mail: pierre.auclair-desrotour@obspm.fr

b e-mail: stephane.mathis@cea.fr

c e-mail: christophe.leponcin@obspm.fr
}

This is an Open Access article distributed under the terms of the Creative Commons Attribution License 4.0, which permits unrestricted use, distribution, and reproduction in any medium, provided the original work is properly cited. 
fication, and their restoring forces are the Coriolis acceleration and the buoyancy respectively. Their characteristic frequencies are the inertial frequency $2 \Omega, \Omega$ being the angular velocity of the body, and the Brunt-Väisälä frequency $N$ corresponding to the radial variation of the specific entropy. Gravitoinertial waves result from their coupling in stably stratified rotating fluid regions. Because of their complexity, studying their dissipation by using a reduced local model is an interesting way to explore its behavior over a large domain of parameters $[11,12]$ and is complementary of works carried out with global models.

The aim of the present work is to propose such a method to investigate the dependence of tidal dissipation on fluid parameters. Using a fluid box in which rotation, stratification, viscosity and thermal diffusivity are taken into account, scaling laws describing the viscous friction applied on tidal waves are obtained. They are used to illustrate, through the concrete example of a planet-satellite system, how the quality factor $Q$ and the evolution of the orbital dynamics are linked to the fluid parameters [10].
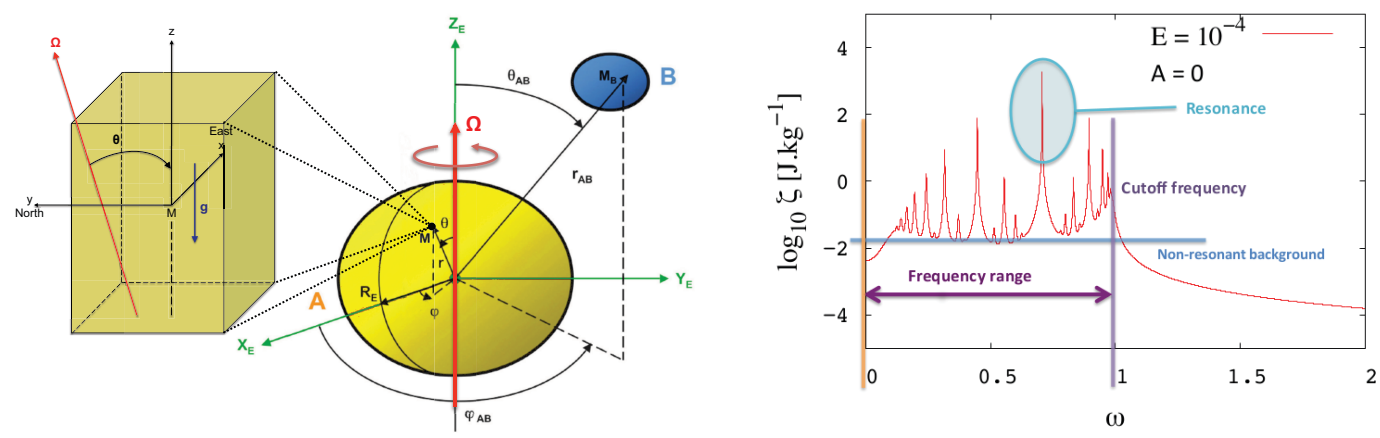

Fig. 1. Left: The local analytical model: a Cartesian fluid section of a rotating fluid body $A$ tidally perturbed by a companion $B$. Right: A dissipation frequency-spectrum computed using the local model, $\zeta$ being the energy dissipated by viscous friction per unit mass in the box over a rotation period. We have introduced the tidal frequency of the perturbation normalized by the inertial frequency (2S), $\omega=\chi / 2 \Omega$, the Ekman number $E$ and the normalized thermal diffusivity $K$ (see their definition in the text). In the case presented here $K=0$ and $A=N / 2 \Omega=0$, which means that the waves are purely inertial and viscously damped (top left blue zone in Fig. 2).

\section{Hydrodynamical scaling laws}

The model used here generalizes the local model presented in [7]. It considers a local section of a fluid region in a rotating celestial body $A$ (of mass $M_{A}$ ) tidally perturbed by a companion $B$ (of mass $M_{B}$ ). It is a rotating Cartesian box of side length $L$ inclined with respect to the spin axis of the body $\boldsymbol{\Omega}$ by a colatidude $\theta$ (Fig. 1, left). The coordinate $z$ corresponds to the radial direction, $x$ and $y$ to the azimutal and latitudinal ones respectively. In the box, the fluid is assumed to be homogeneous of density $\rho$ and to have a kinetic viscosity $v$ and a thermal diffusivity $\kappa$. Three dimensionless control parameters are identified: $A=(N / 2 \Omega)^{2}$ gives the nature of the waves $(A \ll 1$ for inertial waves, $A \gg 1$ for gravity ones), the Ekman number $E=2 \pi^{2} v /\left(\Omega L^{2}\right)$ compares the term of viscous diffusion with respect to the Coriolis one, and $K=2 \pi^{2} \kappa /\left(\Omega L^{2}\right)$ is equivalent to $E$ for thermal diffusion. The Prandlt number $P_{r}=E / K$ compares the viscous and thermal diffusions (see Fig. 2).

Expanding physical quantities into Fourier series in time and space allows us to compute an analytical expression for the energy $\zeta$ dissipated per unit mass by viscous friction over a rotation period $(T=2 \pi / \Omega)$. This gives access to the properties of the dissipation spectrum (Fig. 1, right), which has the shape of a batch of resonances located between $\omega_{-}$and $\omega_{+}$given by [13]. Four asymptotic behaviors are identified. They are represented in Fig. 2 and illustrated by the corresponding spectra. Scaling laws for the viscous friction are derived analytically in each regime for the frequencies $\omega_{m n}$, 

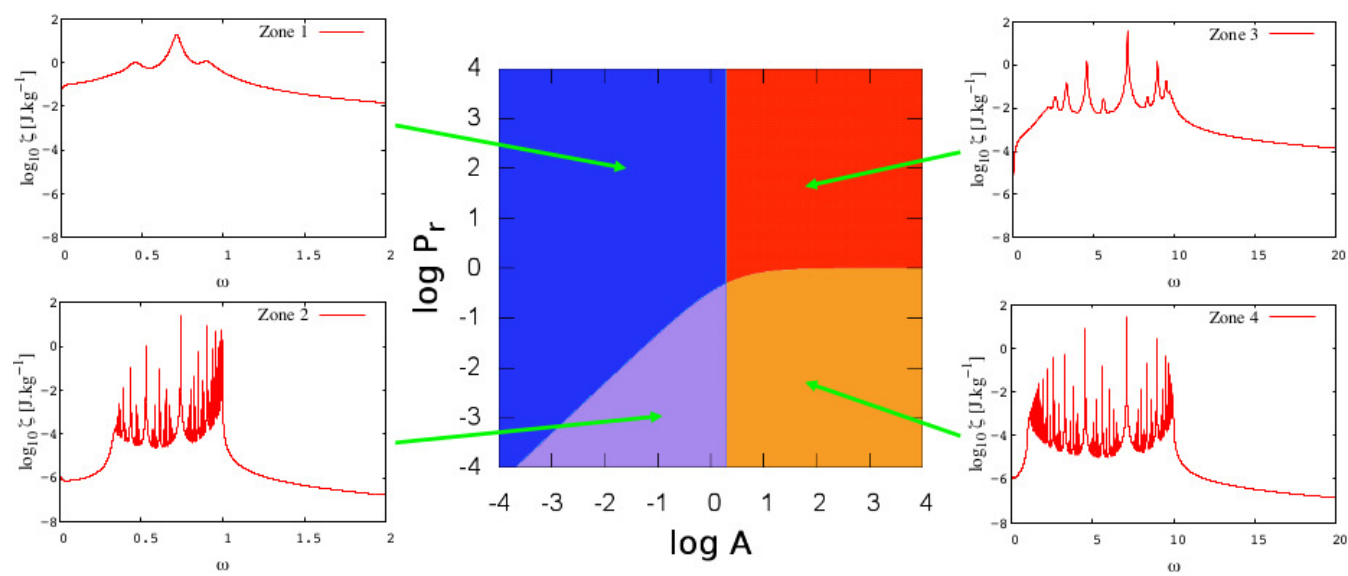

Fig. 2. Asymptotic behaviors of tidal gravito-inertial waves. Zones colored in blue and purple correspond to inertial waves, the two other ones to gravity waves ; zones colored in blue and red correspond to the case where viscous diffusion predominates over thermal diffusion, the two zones below corresponding to the opposite case.

the width at mid-height $l_{m n}$ and height $H_{m n}$ of resonances $\left(\{m, n\} \in \mathbb{Z}^{* 2}\right)$, the number of peaks $N_{\mathrm{kc}}$, the height of the non-resonant background $H_{\mathrm{bg}}$ (which corresponds to the so-called equilibrium tide) and the sharpness ratio $\Xi=H_{11} / H_{\mathrm{bg}}$ of the spectrum that gives the relative contrast between the principal $\{1,1\}$ resonance and the background (Table 1$)$.

Table 1. Scaling laws for the properties of the energy viscously dissipated for the different regimes (we define $A_{11} \equiv 2 \cos ^{2} \theta$ and $\operatorname{Pr}_{11} \equiv A /\left(A+A_{11}\right)$ ). Top left: Inertial waves dominated by viscosity. Top right: Gravity waves dominated by viscosity. Bottom left: Inertial waves dominated by heat diffusion. Bottom right: Gravity waves dominated by heat diffusion. $F$ is the amplitude of the forcing.

\begin{tabular}{l|ll|ll}
\hline \hline DomaIN & $A \ll A_{11}$ & & $A \gg A_{11}$ & \\
\hline $\operatorname{Pr} \gg \operatorname{Pr}_{11}$ & $\frac{\chi_{m n}}{2 \Omega} \propto \frac{n}{\sqrt{m^{2}+n^{2}}} \cos \theta$ & $N_{\mathrm{kc}} \propto E^{-1 / 2}$ & $\frac{\chi_{m n}}{2 \Omega} \propto \frac{m}{\sqrt{m^{2}+n^{2}}} \sqrt{A}$ & $N_{\mathrm{kc}} \propto A^{1 / 4} E^{-1 / 2}$ \\
& $l_{m n} \propto E$ & $H_{m n} \propto F^{2} E^{-1}$ & $l_{m n} \propto E$ & $H_{m n} \propto F^{2} E^{-1}$ \\
& $H_{\mathrm{bg}} \propto F^{2} E$ & $\Xi \propto E^{-2}$ & $H_{\mathrm{bg}} \propto F^{2} E A^{-1}$ & $\Xi \propto A E^{-2}$ \\
\hline $\operatorname{Pr} \ll \operatorname{Pr}_{11}$ & $\frac{\chi_{m n}}{2 \Omega} \frac{n}{\sqrt{m^{2}+n^{2}}} \cos \theta$ & $N_{\mathrm{kc}} \propto A^{-1 / 2} K^{-1 / 2}$ & $\frac{\chi_{m n}}{2 \Omega} \propto \frac{m}{\sqrt{m^{2}+n^{2}}} \sqrt{A}$ & $N_{\mathrm{kc}} \propto A^{1 / 4} K^{-1 / 2}$ \\
& $l_{m n} \propto A K$ & $H_{m n} \propto F^{2} A^{-2} E K^{-2}$ & $l_{m n} \propto K$ & $H_{m n} \propto F^{2} E K^{-2}$ \\
& $H_{\mathrm{bg}} \propto F^{2} E$ & $\Xi \propto A^{-2} K^{-2}$ & $H_{\mathrm{bg}} \propto F^{2} E A^{-1}$ & $\Xi \propto A K^{-2}$ \\
\hline \hline
\end{tabular}

\section{Impact on tidal dynamics}

The properties of the dissipation directly impact the long-term evolution of planetary systems. This point is easily illustrated through the example of a two-body coplanar circular planet-moon system [14]. Indeed, if we introduce in the dynamical equations a frequency-dependent tidal quality factor [15] proportional to the inverse of $\zeta$ computed with our local model, the semi-major axis $a$ of the system evolves erratically (Fig. 3). Instead of falling on the planet regularly with an increasing velocity 
as in the case corresponding to Kaula's constant $Q$ model [16], the satellite only comes nearer to it, jumping from a position to an other each times it meets a peak of resonance. Under some assumptions detailed in [10], the relative amplitude of a jump $\Delta a / a$ can be written as a function of the frequency $\omega_{p}$, of the width at mid-height $l_{p}$ and of the sharpness ratio $\Xi_{p}=H_{p} / H_{\mathrm{bg}}$ of a peak,

$$
\frac{\Delta a}{a} \approx 2 l_{\mathrm{p}}\left[3 \sqrt{\sqrt{2}-1}\left(1+\omega_{\mathrm{p}}\right)\right]^{-1}\left[\sqrt{\Xi_{p}}-1\right]^{\frac{1}{2}} .
$$

Each of these characteristics are now given explicitly as functions of the parameters of the fluid, $A, E$ and $K$ thanks to scaling laws obtained in Table 1 .
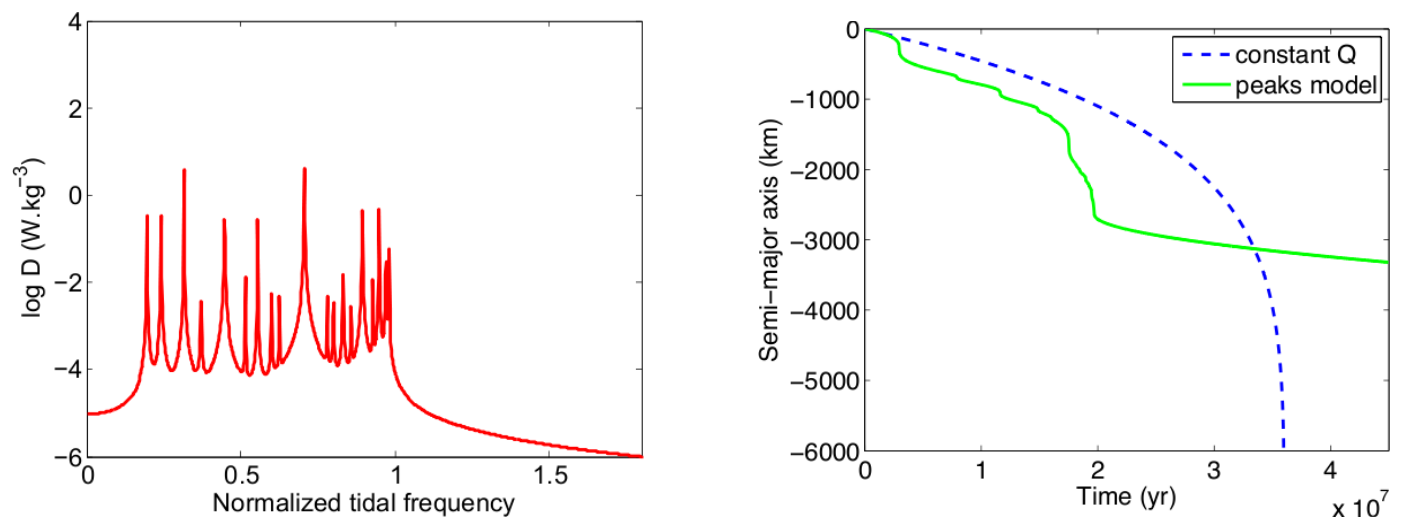

Fig. 3. Left: A synthetical dissipation spectrum generated using the local model of the fluid box; $D$ is proportional to $\zeta$ computed for $E=10^{-5}$. Right: The corresponding evolution of the semi-major axis $a$ of the fluid planetsatellite system as a function of time [10].

\section{Conclusions}

The properties of fluid tidal dissipation have a direct impact on the secular dynamics of planetary systems. Indeed, dissipation resonances cause local rapid changes of orbital parameters, that are directly related to the properties of the corresponding peaks. In this context, our local model provides scaling laws that describe the evolution of the complex and resonant tidal dissipation as a function of fluid parameters. In a near future, our method could be extended to magnetized fluid regions. This will contribute to improve tidal dissipation modeling in studies of the dynamical evolution of planetary systems.

\section{References}

1. P. Goldreich, S. Soter, Icarus 5, 375 (1966)

2. J. Laskar, G. Boué, A.C.M. Correia, A\&A 538, A105 (2012), 1110. 4565

3. E. Bolmont, S.N. Raymond, P. von Paris, F. Selsis, F. Hersant, E.V. Quintana, T. Barclay, ApJ 793, 3 (2014), 1404.4368

4. M. Efroimsky, ApJ 746, 150 (2012), 1105. 3936

5. F. Remus, S. Mathis, J.P. Zahn, V. Lainey, A\&A 541, A165 (2012), 1204.1468

6. J.P. Zahn, A\&A 41, 329 (1975)

7. G.I. Ogilvie, D.N.C. Lin, ApJ 610, 477 (2004), arXiv: astro-ph/0310218

8. G.I. Ogilvie, D.N.C. Lin, ApJ 661, 1180 (2007), astro-ph/0702492 
9. F. Remus, S. Mathis, J.P. Zahn, A\&A 544, A132 (2012), 1205. 3536

10. P. Auclair-Desrotour, C. Le Poncin-Lafitte, S. Mathis, A\&A 561, L7 (2014), 1311.4810

11. G.I. Ogilvie, Journal of Fluid Mechanics 543, 19 (2005), astro-ph/0506450

12. L. Jouve, G.I. Ogilvie, Journal of Fluid Mechanics 745, 223 (2014), 1402 . 2769

13. T. Gerkema, V.I. Shrira, Journal of Fluid Mechanics 529, 195 (2005)

14. M. Efroimsky, V. Lainey, Journal of Geophysical Research (Planets) 112, E12003 (2007), 0709. 1995

15. S. Mathis, C. Le Poncin-Lafitte, A\&A 497, 889 (2009)

16. W.M. Kaula, Reviews of Geophysics and Space Physics 2, 661 (1964) 